\section{MATERIALS SCIENCE}

\section{Conductors with a twist}

Nature Mater. 8, 421-426 (2009)

Discotic liquid crystals are flat-cored organic molecules that stack in twisting, electronconducting columns. They are used in photovoltaics and field-effect transistors. The best discotic species currently in use make stacks with a twist angle of $30^{\circ}$, but calculations show $60^{\circ}$ to be optimal for conduction.

Klaus Müllen and Denis Andrienko of the Max Planck Institute for Polymer Research in Mainz, Germany, and their colleagues have synthesized a new molecule with a $60^{\circ}$ twist. This doubled electron mobility, and through molecular dynamics simulations, the authors show that removing defects in the stacking structure could push that value higher.

\section{COSMOLOGY}

\section{No ring or reason}

Astrophys. J. 696, 694-700 (2009)

A new study raises questions about the ring of dark matter reported to exist inside a galaxy cluster.

The cluster, called Cl0024+17, is actually thought to be two merging clusters, and some suggest that the ring was caused by their collision. To test the idea, John ZuHone, now at the Harvard-Smithsonian Center for Astrophysics in Cambridge, Massachusetts, and his colleagues simulated cluster collisions under varying conditions. They could not produce a ring unless the dark-matter particles had circular orbits.

Because such orbits are unlikely to occur in galaxy clusters, the results suggest that the ring report is questionable, says $\mathrm{ZuHone}$.

\section{PLANT PHYSIOLOGY}

\section{Gifts from grafts}

Science 324, 649-651 (2009)

Plants grafted together exchange genetic information, suggesting a new and surprising mechanism for gene transfer between organisms.

Grafting is commonly used in cultivation and can occur naturally when shoots or roots from different trees come into contact, but grafting was not thought to involve any mixing of genetic material. To test this, Sandra Stegemann and Ralph Bock of the Max Planck Institute for Molecular Plant Physiology in Potsdam-Golm, Germany, grafted together two transgenic tobacco plants expressing different antibiotic-resistance genes.

The resistance genes were frequently exchanged between cells across the graft site. However, transfer only occurred when the genes were carried in the chloroplast genome, not when a resistance gene was inserted into the nuclear genome. Because the genetic exchange was limited to the graft site, the genes would only be passed to offspring of shoots formed at that site.

\section{STRUCTURAL BIOLOGY}

\section{A virus laid bare}

PLoS Biol. 7, e1000092 (2009)

Mimivirus was once mistaken for a

bacterium owing to its shape and great size.

Michael Rossmann of Purdue University in

West Lafayette, Indiana, and his colleagues

have now revealed the structure of this largest

of known viruses. Enzymatic scrubbing

of long fibres that extend from the viral

capsid gave the researchers a new view. They reconstructed the icosahedral shape of the virus using cryogenic electron microscopy and atomic force microscopy.

Mimivirus is not as symmetrical as many other large, double-stranded DNA viruses, in part because of a 'starfish' feature that appears on one side. This feature may allow the virus to deliver its genome into host cells.

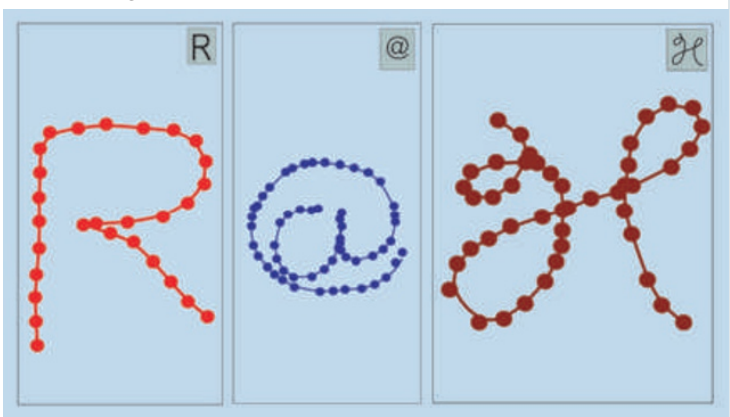

\section{NANOTECHNOLOGY}

\section{The helix that delivers}

Nano Lett. 10.1021/nl900186w (2009)

For nanomedical applications such as delivering drugs or performing microsurgical procedures at specific places in the body, the ability to navigate bodily fluids with a high degree of control is crucial.

With this in mind, Ambarish Ghosh and Peer Fischer of the Rowland Institute at Harvard produced screw-like glass structures 1-2 micrometres long by nanofabrication. Depositing a thin layer of a ferromagnetic material on one side of each helix allowed the authors to direct the screws' motion with rotating magnetic fields. The duo even used the swimmers to spell out the initials of their institution (pictured above).

The microswimmers can carry chemicals and push loads, and could be used as probes in rheological measurements difficult to perform by other techniques.

\section{JOURNAL CLUB}

\section{David Kirchman University of Delaware, Lewes}

\section{A microbial ecologist learns something new from an old- fashioned study.}

What could be easier than learning about an organism simply by watching how it varies over time in its natural habitat? You'd think this would have been done long ago for marine bacteria, which are important in many biogeochemical processes, including the carbon cycle; in fact, they're the organisms running the biosphere. But it's not easy to follow microbes in the open ocean, far from the lab and beyond the reach of standard techniques.

Craig Carlson at the University of California, Santa Barbara, and his colleagues took on this challenge for the most abundant group of marine bacteria: SAR11. They examined variations in SAR11 over several years in the Sargasso Sea, where the group was first discovered nearly 20 years ago (C. A. Carlson et al. ISME J. 3, 283-295; 2009). Sequencing and other data had previously revealed that SAR11 bacteria are diverse and can account for almost $50 \%$ of microbes in a given marine environment; however, we still knew little about their natural history.

So Carlson's group looked to address a basic question: how do different members of SAR11 vary with depth and over time? They examined 13 years' worth of DNA samples, viewed 3 years' worth of preserved cells under the microscope, and then analysed the microbial data in light of what is known about SAR11's environment. Three SAR11 'ecotypes', they say, flourish differently at various depths and over a yearly cycle, which starts in spring, when deep mixing stops and photosynthesis speeds up.

The authors make good use of new genomic data from a labgrown representative of SAR11 (Pelagibacter ubique) to understand Sargasso Sea populations, but the study's insight comes from the old approach of patiently watching organisms over time in their natural habitat.

Discuss this paper at http://blogs. nature.com/nature/journalclub 